\title{
PanK4 inhibits pancreatic $\beta$-cell apoptosis by decreasing the transcriptional level of pro-caspase-9
}

\author{
Ruo Lan Xiang, ${ }^{1, *}$, Yan Li Yang ${ }^{1, *}$, Jin Zuo ${ }^{1}$, Xin Hua Xiao ${ }^{2}$, Yong Sheng Chang ${ }^{1}$, Fu De Fang ${ }^{1}$ \\ ${ }^{I}$ National Laboratory of Medical Molecular Biology, Institute of Basic Medical Sciences, Peking Union Medical College \& Chinese \\ Academy of Medical Sciences, 5\# Dong Dan San Tiao, Beijing 100005, China; ${ }^{2}$ Peking Union Medical College Hospital, Beijing \\ 100730, China
}

Cell Research (2007) 17:966-968. doi: 10.1038/cr.2007.93; published online 13 November 2007

\section{Dear Editor:}

Coenzyme $\mathrm{A}(\mathrm{CoA})$ is a primary and predominant acyl group carrier involved in a wide variety of important biochemical processes. The CoA biosynthetic pathway is composed of five enzymatic steps, of which Pantothenate kinase (PanK) is a key regulatory enzyme. The multiple isoforms of PanK are encoded by four different genes [1,2]. In our previous studies of SNP markers by genotyping the case-controlled DNAs, we found that one SNP within the hPANK4 gene on chromosome 1 was associated with type 2 diabetes [3-5]. We subsequently showed that rat PanK4 (rPanK4) was up-regulated when rats were challenged by high concentration of glucose [6]. M2-type pyruvate kinase (Pkm2) was found, both in vitro and in vivo, to be associated with rPanK4 [7]. These data suggest that PanK4 may have a role in diabetes pathogenesis. The development of type 2 diabetes is due partly to the loss of the pancreatic $\beta$-cell mass, therefore the secreted amount of insulin is insufficient to maintain the glucose homoestasis [8]. In the present study, we evaluated the effect of rPanK4 on $\beta$-cell apoptosis. We aimed to determine the potential of rPanK4 gene in $\beta$-cell apoptosis induced by the cytotoxic agent streptozotocin (STZ).

We generated a rat RINm5F cell line (insulin-secreting tumor cell line) overexpressing recombinant rPanK4 (rPanK4-RINm5F). Cells transfected with empty vector (empty-RINm5F) were used as the negative control (Fig-

\footnotetext{
* These authors contributed equally to this work. Correspondence: Fu De Fang ${ }^{1}$, Yong Sheng Chang ${ }^{2}$

${ }^{1}$ Tel/Fax: +86 010 65253005; E-mail: fangfd@vip.sina.com

${ }^{2}$ Tel: +86 010 65296424; Fax: +86 01065253005 ; E-mail: changyongsheng@ yahoo.com
}

ure 1A). We examined the effects of rPanK4 on cellular proliferation by MTT assay. Data shows that there were little differences in rates of cellular proliferation comparing the control and rPanK4-RINm5F cells. Accordingly, we focused on the potential role of rPanK4 in apoptosis process. Here, we used FACS to investigate the role of rPanK4 in STZ-induced apoptosis. RINm5F cells were exposed to a series of concentrations of STZ at the different time points. The apoptosis rate of rPanK4-RINm5F cells was significantly reduced compared to control cells only when cells were exposed to $2.5 \mathrm{mg} / \mathrm{ml}$ of STZ for $24 \mathrm{~h}$. When RINm5F and empty-RINm5F transfected cells were exposed to $2.5 \mathrm{mg} / \mathrm{ml}$ of STZ, the apoptosis rate increased from $6.1 \pm 0.7 \%$ to $19.5 \pm 0.9 \%$ and from $7.3 \pm 0.4 \%$ to $20.3 \pm 0.7 \%$ respectively. By contrast, the apoptosis rate of the rPanK4-RINm5F cells only slightly increased to $11.1 \pm$ $1.2 \%(p<0.01)$ (Figure 1B1). The rPanK4 protective effect was further evaluated by using TUNEL and DNA ladder assays. The apoptosis (Figure 1B2, B4, B5, B6) and DNA fragmentation (Figure 1B3) were significantly decreased in rPanK4-overexpressing cells when exposed to $2.5 \mathrm{mg} / \mathrm{ml}$ of STZ for $24 \mathrm{~h}$.

We further examined the effects of rPanK4 on apoptosisrelated genes by quantitative real-time PCR. Overexpression of $\mathrm{rPanK} 4$ significantly reduced the expression level of the pro-caspase- 9 mRNA by more than $50 \%$, but not other apoptosis-related genes, including Bcl-2, Bax, Bad, pro-caspase- 3 , and pro-caspase- 8 genes (Figure 1C). At protein level, pro-caspase- 9 was significantly decreased in $\mathrm{rPanK} 4-\mathrm{RINm} 5 \mathrm{~F}$ cells. However, the pro-caspase-3 expression levels were not significantly different between rPanK4-RINm5F and RINm5F cells (Figure 1D1).

We analyzed the caspase activation by colorimetric and western blot assays. Caspase- 3 and caspase- 9 activities 
$\mathrm{A}_{(\mathrm{A} 1)}$

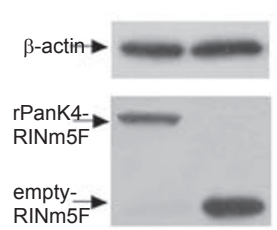

B

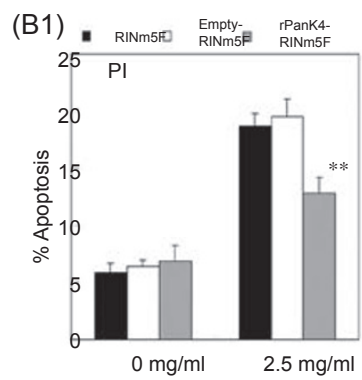

(B3)
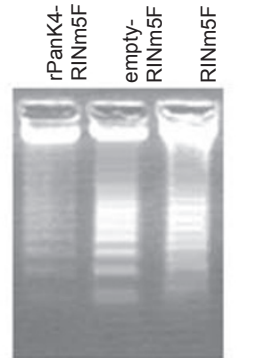

(B5)

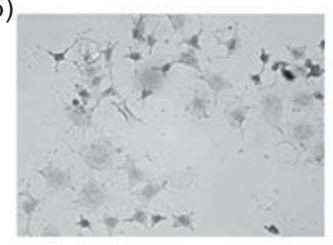

Empty-RINm5F
(A2)

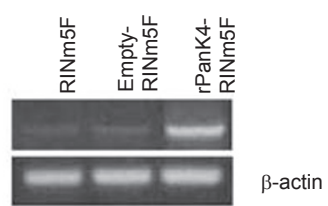

(B2)

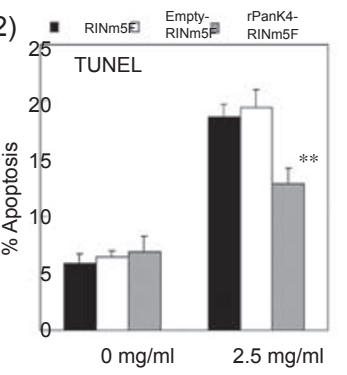

(B4)

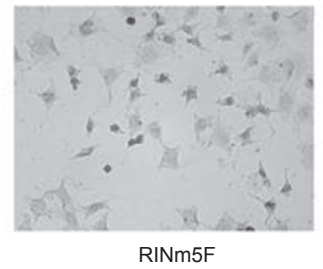

(B6)

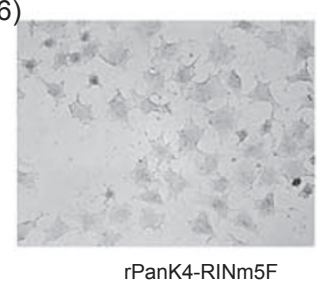

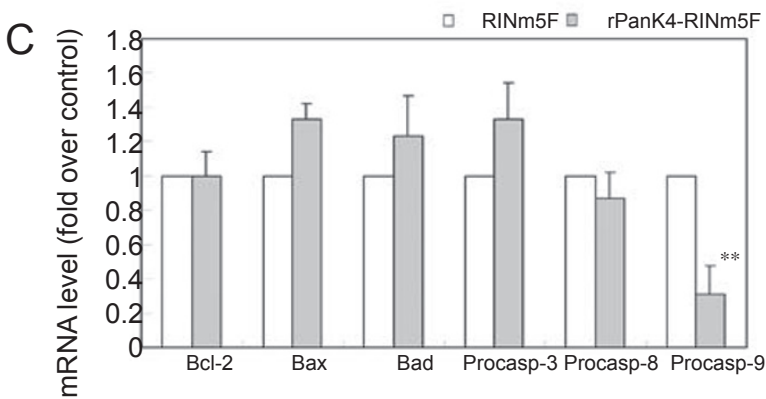

D

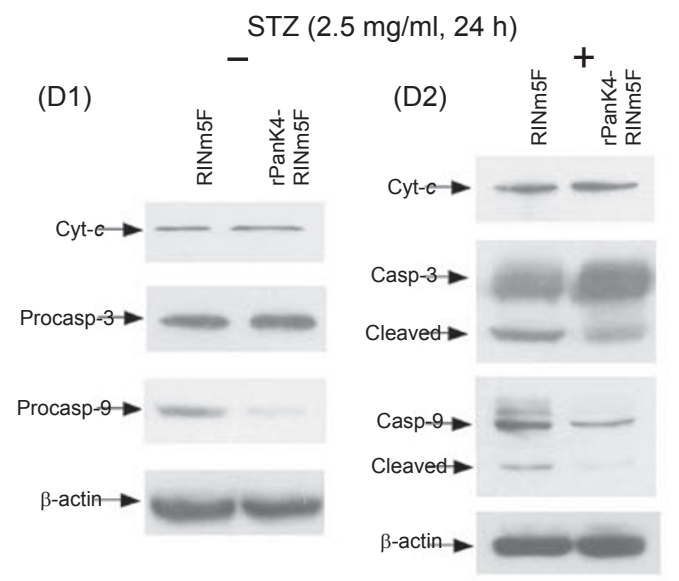

(D3)

(D4)
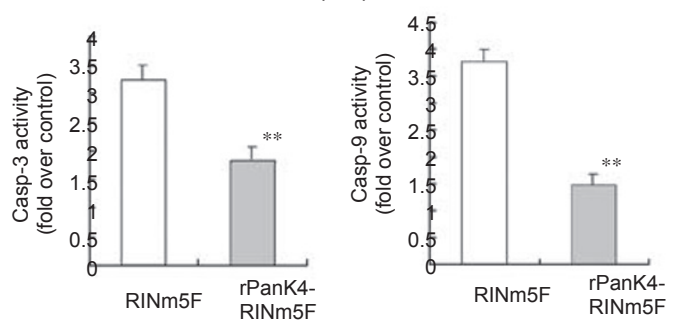

Figure 1 (A) Stable expression of rPanK4 in RINm5F cells. (A1) Western blot of EGFP protein (27 kDa) expressed in empty-vector transfected cells, and pEGFP-rPanK4 fusion protein $(103 \mathrm{kDa})$ in cells transfected with the rPanK4-pEGFP vector. (A2) RT-PCR detection of rPanK4 in RINm5F cells. (B) Effects of rPanK4 on STZ-induced apoptosis. (B1) Apoptotic cells labeled with PI were quantified by FACS. (B2) The summary of apoptosis detected by the TUNEL assay. (B3) Detection of apoptotic cells by DNA ladder assay. (B4, 5, 6) Representative microscopic views of apoptosis after the TUNEL staining $(\times 400)$. (C) Real-time PCR detection of apoptosis-related genes. (D) Western blotting of apoptosis-related proteins. (D1, 2) Western blotting of cytochrome $c$, caspase-9 and caspase-3. (D3) Detection of caspase-3 activity. (D4) Detection of caspase-9 activity. ${ }^{* *} p<0.01$ compared with control by using unpaired Students' $t$-test.

were found decreased in rPanK4-RINm5F cells compared to RINm5F cells stimulated with STZ (Figure 1D2, D3, D4). Taken together, it might imply that overexpression of rPanK 4 partially inhibited the caspase- 3 and caspase9 activities in STZ-induced apoptosis in RINm5F cells. Moreover, the decrease in the caspase- 3 activity was correlated with the decreased caspase- 9 activity.

To test whether the decrease of caspase- 9 activity was due to the changes of cytochrome $c$, we compared the level of cytochrome $c$ in cytosol in RINm5F and rPanK4RINm5F cells by western blot. Figure 1D shows that the level of cytochrome $c$ in rPanK4-RINm5F cells was similar to that in the control cells. The result of cytosol cytochrome $c$ also suggested that overexpressing rPanK4 had little effect on the mitochondrial function.

In our previous studies, PanK4 was shown to be related to diabetes. Interestingly, PanK 4 does not possess a kinase activity and thus does not change cell proliferation/apop- 
tosis via its activity in CoA metabolism [9]. Evidence with the Drosophila orthologue of PanK gene fumble $(f b l)$ suggested a CoA-independent role in cell proliferation [10]. We showed that STZ-stimulated apoptosis was significantly blocked by an overexpression of rPanK4. In the present study, rPanK4 expression led to decreased pro-caspase- 9 mRNA level. Accordingly, the expression of pro-caspase- 9 protein also decreased. Consistently, rPanK4-overexpressing cells underwent significantly lower caspase-3 activation when treated with STZ.

In conclusion, our current study suggests that rPanK4 has an anti-apoptotic effect, via down-regulating caspase-9. Given this novel finding, future studies are needed to elucidate the biological functions and molecular mechanisms of PanK4 in type 2 diabetes.

\section{Acknowledgments}

We are indebted to Dr Jin Ming Gao for critical reading of this manuscript. This work was supported by the National Basic Research Program of China (2004CB518602 and 2006CB503909), the National Natural Science Foundation of China (30471930) and the Beijing Natural Science Foundation (5072042).

\section{References}

1 Jackowski S, Rock CO. Regulation of coenzyme A biosynthesis.
J Bacteriol 1981; 148:926-932.

2 Rock CO, Calder RB, Karim MA, Jackowski S. Pantothenate kinase regulation of the intracellular concentration of coenzyme A. J Biol Chem 2000; 275:1377-1383.

3 Zhao JY, Xiong MM, Huang W, et al. An autosomal genomic scan for loci linked to type 2 diabetes in northern Han Chinese. J Mol Med 2005; 83:209-215.

4 Li Y, Wu GD, Zuo J, et al. Screening susceptibility genes of type 2 diabetes in Chinese population by single nucleotide polymorphism analysis. Zhongguo Yi Xue Ke Xue Yuan Xue Bao. 2005; 27:274-279.

5 Li Y, Wu G, Zuo J, Gao J, Chang Y, Fang FD. Genetic variations of the CDC2L2 gene are associated with type 2 diabetes in a Han Chinese cohort. Diabetes Metab Res Rev 2007; 23:455-461.

6 Chang YS, Li YF, Zuo J, Fang FD. A novel gene involved in blood glucose regulation. Zhongguo Yi Xue Ke Xue Yuan Xue Bao 2002; 24:466-470.

7 Li YF, Chang YS, Zhang LF, et al. High glucose upregulates pantothenate kinase4 (PANK4) thus affectes M2-type pyrvate kinase (Pkm2). Mol Cell Biochem 2005; 277: 117-125.

8 Butler AE, Janson J, Bonner-Weir S, Ritzel R, Rizza RA, Butler PC. Beta-cell deficit and increased beta-cell apoptosis in humans with type 2 diabetes. Diabetes 2003; 52:102-110.

9 Zhang YM, Chohnan S, Virga KG, et al. Chemical Knockout of Pantothenate Kinase Reveals the Metabolic and Genetic Program Responsible for Hepatic CoenzymeA Homeostasis. Chem \& Biol 2007; 14:291-302.

10 Afshar K, Gonczy P, DiNardo S, Wasserman SA. Fumble encodes a pantothenate kinase homolog required for proper mitosis and meiosis in Drosophila melanogaster. Genetics 2001; 157:12671276. 